\title{
Central Pain Due to Spinothalamic Tract Injury by Head Trauma Caused by Falling Object
}

\author{
Sung Ho Jang, MD, You Sung Seo, MS
}

Department of Physical Medicine and Rehabilitation, Yeungnam University College of Medicine, Daegu, Korea

In this report, we described the case of a 54-year-old female who suffered trauma from a falling large box (100 $\mathrm{cm} \times 30 \mathrm{~cm} \times 30 \mathrm{~cm}$ ) that hit the vertex of her head while seated in a subway car. The patient experienced loss of consciousness for several minutes, and post-traumatic amnesia for several minutes, with a Glasgow Coma Scale score of 15. She experienced severe bursting pain in her head, neck and upper back immediately after the head trauma; and approximately 3 hours later, she began to feel pricking pain in her left arm and leg. Despite visits to several hospitals to determine the cause of pain, no specific diagnosis was confirmed. Ten months postinjury, she visited the Department of Rehabilitation of a Yeunnam University Hospital with the chief complaint of constant pain with hyperalgesia in her head, left arm, and leg. The characteristics and severity of pain were as follows: head, pricking sensation (visual analogue scale [VAS], 5-7]; left arm, pricking and squeezing (VAS, 3-7); and left leg, bursting sensation (VAS, 6-7). The subscales of Nottingham Sensory Assessment indicated normal kinesthetic sensation score of the left extremities (24 out of a possible 24 points), but impaired tactile sensation score (10 out of a possible 20 points). Brain and spine magnetic resonance imaging revealed no specific focal lesion; additionally, an electromyography study showed no evidence of peripheral neuropathy or radiculopathy.
Diffusion tensor imaging data were acquired at 10 months post-injury using a 6 -channel head coil on a $1.5 \mathrm{~T}$ Philips Gyroscan Intera (Philips, Best, Netherlands). The Oxford Centre for Functional Magnetic Resonance Imaging of the Brain (FMRIB) Software Library (FSL; www. fmrib.ox.ac.uk/fsl) was used to analyze the diffusionweighted imaging data. Fiber tracking was performed using a probabilistic tractography method based on a multifiber model. The spinothalamic tracts (STTs) were located by selection of fibers passing through regions of interest (ROIs). A seed ROI was placed on the posterolateral medulla on an axial slice and a target ROI was placed on the primary somatosensory cortex on an axial slice. The threshold of 1 streamline was applied for the results of fiber tracking [1]. Ten-month diffusion tensor tractography indicated partial tearing and narrowing of the STTs in both hemispheres (Fig. 1B). The patient was prescribed gabapentin (900 mg/day) for 1 month, which resulted in partial pain control to a tolerable level.

In this study, analysis of the STT in a patient with central pain following head trauma by a falling object indicated partial injury of the STTs in both hemispheres. The cause of central pain and traumatic axonal injury were the most likely pathogenetic mechanisms for the STT injuries [2-4]. 

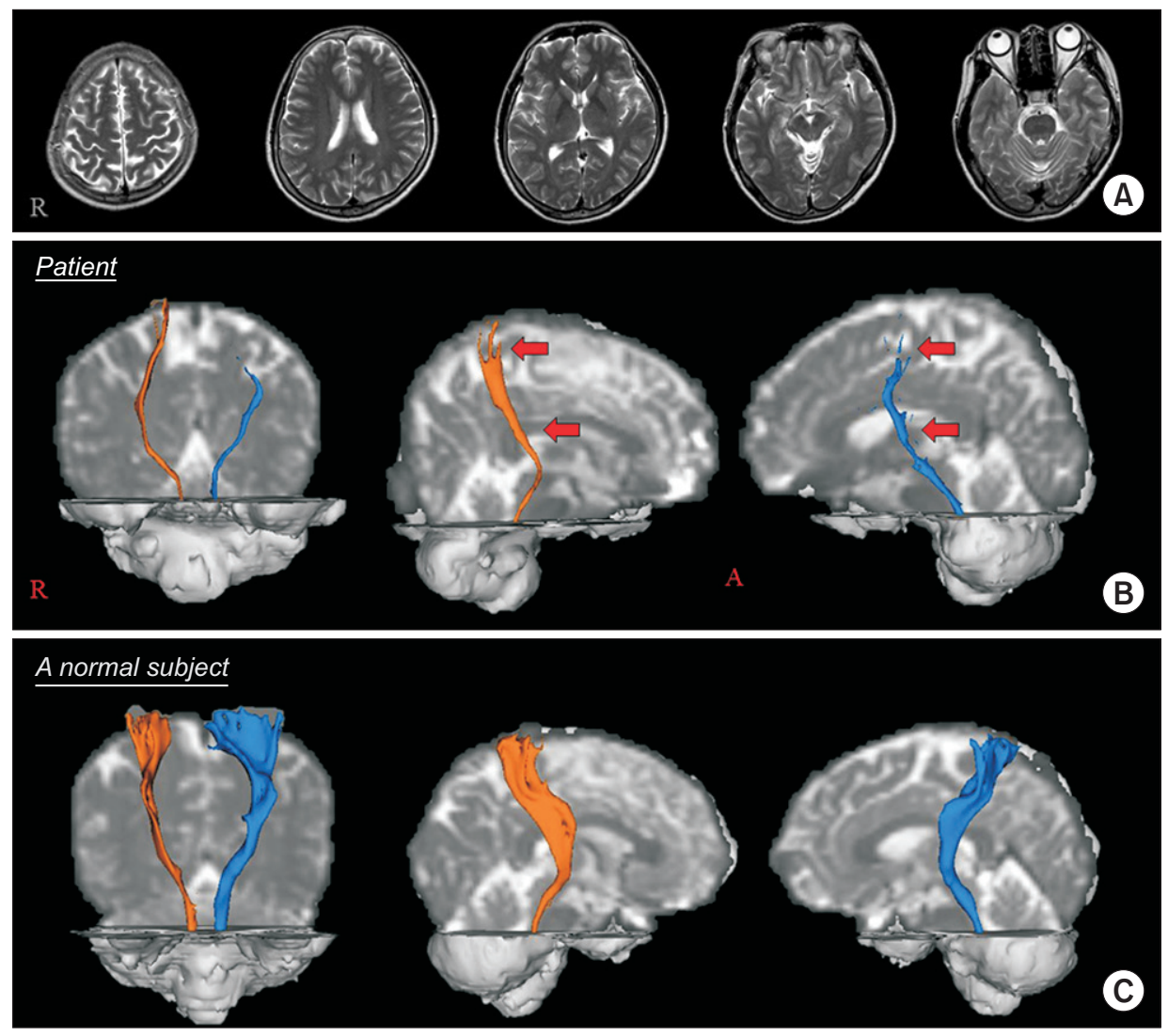

Fig. 1. (A) T2-weighted brain magnetic resonance images at 10 months after head trauma show no abnormal lesion. (B) Diffusion tensor tractography for the spinothalamic tract show partial tearing and narrowing of the spinothalamic tracts in both hemispheres, as compared with those of a normal subject (53-year-old female).

\section{CONFLICT OF INTEREST}

No potential conflict of interest relevant to this article was reported.

\section{ACKNOWLEDGMENTS}

This work was supported by the National Research Foundation (NRF) of Korea Grant funded by the Korean Government (MSIP) (2015R1A2A2A01004073).

\section{REFERENCES}

1. Kim JH, Ahn SH, Cho YW, Kim SH, Jang SH. The relation between injury of the spinothalamocortical tract and central pain in chronic patients with mild traumatic brain injury. J Head Trauma Rehabil 2015;30:E40-6.

2. Alexander MP. Mild traumatic brain injury: pathophysiology, natural history, and clinical management. Neurology 1995;45:1253-60.

3. Povlishock JT, Christman CW. The pathobiology of traumatically induced axonal injury in animals and humans: a review of current thoughts. J Neurotrauma 1995;12:555-64.

4. Buki A, Povlishock JT. All roads lead to disconnection? Traumatic axonal injury revisited. Acta Neurochir (Wien) 2006;148:181-94. 